\title{
Self-avoidance and Tiling: Mechanisms of Dendrite and Axon Spacing
}

\author{
Wesley B. Grueber ${ }^{1}$ and Alvaro Sagasti ${ }^{2}$ \\ ${ }^{1}$ Department of Physiology and Cellular Biophysics, Department of Neuroscience, Columbia University \\ Medical Center, New York, New York 10032 \\ ${ }^{2}$ Department of Molecular, Cell, and Developmental Biology, University of California, Los Angeles, \\ Los Angeles, California 90095 \\ Correspondence: sagasti@mcdb.ucla.edu andwg2135@columbia.edu
}

The spatial pattern of branches within axonal or dendritic arbors and the relative arrangement of neighboring arbors with respect to one another impact a neuron's potential connectivity. Although arbors can adopt diverse branching patterns to suit their functions, evenly spread branches that avoid clumping or overlap are a common feature of many axonal and dendritic arbors. The degree of overlap between neighboring arbors innervating a surface is also characteristic within particular neuron types. The arbors of some populations of neurons innervate a target with a comprehensive and nonoverlapping "tiled" arrangement, whereas those of others show substantial territory overlap. This review focuses on cellular and molecular studies that have provided insight into the regulation of spatial arrangements of neurite branches within and between arbors. These studies have revealed principles that govern arbor arrangements in dendrites and axons in both vertebrates and invertebrates. Diverse molecular mechanisms controlling the spatial patterning of sister branches and neighboring arbors have begun to be elucidated.

\begin{abstract}
A xonal and dendritic arbors adopt complex Aand morphologically diverse shapes that influence neural connectivity and information processing. In this article we review anatomical and molecular studies that elucidate how the arrangements of branches within neuronal arbors are established during development (isoneuronal spacing) and how the relative spacing of arbors is determined when multiple neurons together innervate a defined territory (heteroneuronal spacing). Together these mechanisms ensure that arbors achieve functionally appropriate coverage of input or output territories.
\end{abstract}

Isoneuronal and heteroneuronal processes display a variety of spacing arrangements, suggesting a diversity of underlying molecular mechanisms. Self-avoidance can occur between branches that arise from a single soma (Yau 1976; Kramer and Kuwada 1983; Kramer and Stent 1985), implying that neurons are able to discriminate "self," which they avoid, from "nonself" arbors, with which they coexist (Kramer and Kuwada 1983). Similarly, arbors from different cells that share the same function and together innervate a defined territory can create a pattern of minimally overlapping neighboring dendritic or axonal fields, known

Editors: Marc Tessier-Lavigne and Alex L. Kolodkin

Additional Perspectives on Neuronal Guidance available at www.cshperspectives.org

Copyright (C) 2010 Cold Spring Harbor Laboratory Press; all rights reserved; doi: 10.1101/cshperspect.a001750

Cite this article as Cold Spring Harb Perspect Biol 2010;2:a001750 
W.B. Grueber and A. Sagasti

as tiling. Such spacing mechanisms ensure that arbors maximize their spread across a territory while minimizing the redundancy with which the territory is innervated. In contrast, adhesive interactions between arbors can operate to maintain coherence of dendrites at specific targets (Zhu and Luo 2004), or to bundle functionally similar processes and possibly coordinate their activity (Campbell et al. 2009). Understanding how processes are patterned relative to one another can help to uncover the functional logic of neural circuit organization.

Here we focus primarily on mechanisms of isoneuronal and heteroneuronal avoidance that result in complete and nonredundant innervation of sensory or synaptic space. Such mechanisms have been studied extensively in systems where neuronal arbors innervate a two-dimensional plane, such as the retina or body wall (Wassle et al. 1981; Perry and Linden 1982; Hitchcock 1989; Lin and Masland 2004; Fuerst et al. 2009; Kramer and Stent 1985; Grueber et al. 2003; Sugimura et al. 2003; Sagasti et al. 2005). However, the principles regulating process spacing in these regions likely also apply in three dimensions, most prominently where processes are segregated into nonoverlapping domains or columns (Huckfeldt et al. 2009). It is also notable that nonneuronal cell types might similarly engage in selfavoidance and form tiling arrangements, including leech comb cells (Jellies and Kristan 1991) and mammalian astrocytes (Bushong et al. 2002; Ogata and Kosaka 2002; Livet et al. 2007). Elucidating the mechanisms of process spacing during development is therefore relevant for understanding principles of tissue organization inside and outside of the nervous system.

\section{CELLULAR MECHANISMS OF ISONEURONAL SPACING}

Sister Branches of an Arbor Occupy Mutually Exclusive Territories

Sister branches of axonal or dendritic arbors that innervate a surface often avoid overlapping with one another (Fig. 1A,B). Minimizing branch overlap allows arbors to innervate more

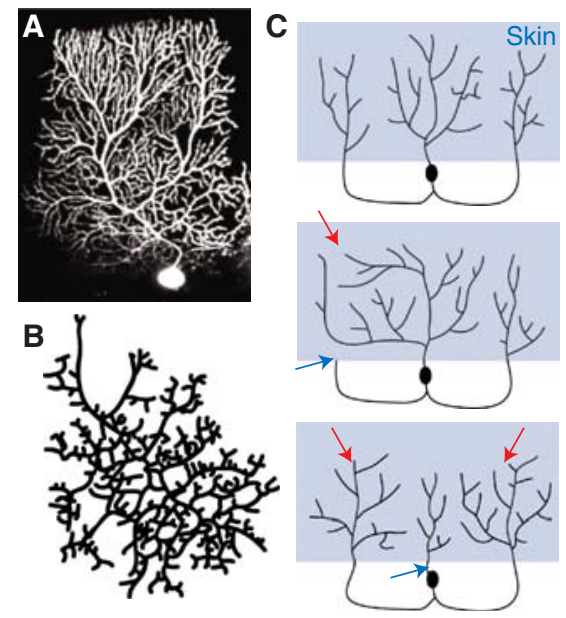

Figure 1. Isoneuronal neurites repel one another. $(A)$ The branches of a mouse cerebellar Purkinje cell dendritic arbor, imaged with 2-photon microscopy in a live animal, almost never overlap with one another (image courtesy of Anna Dunaevsky, Brown University). (B) The branches of a retinal ganglion cell in culture almost never overlap with one another. (Image modified from Montague and Friedlander 1991). (C) Subfields of leech mechanosensory axons compete for territory. Simplified diagrams, based on results in (Kramer and Stent 1985). The top shows a normal leech mechanosensory neuron with three separate subfields innervating adjacent regions of the epidermis. When the growth cone of one branch is crushed (blue arrows in middle and bottom panels), either eliminating (middle) or delaying (bottom) its growth, sibling subfields grow correspondingly larger (red arrows).

territory, and perhaps avoid receiving redundant inputs. Even neurons in culture retain their minimally overlapping character, demonstrating that crossover avoidance can arise through cell-autonomous processes (Montague and Friedlander 1989; Montague and Friedlander 1991). In principle, minimal branch overlap could be achieved with a genetically determined prepattern, a regulated pruning process that eliminates overlapping branches, or competitive interactions between branches during the arborization process. Leech mechanosensory neurons that innervate the epidermis were an early model for elucidating the cellular mechanisms that regulate isoneuronal branch arrangement, and studies in this system have 
revealed several fundamental principles of spacing control. These neurons possess a stereotyped axon arbor architecture consisting of multiple subfields that innervate adjacent parts of the epidermis (Fig. 1C). Each subfield itself occupies a defined territory that does not overlap with other subfields of the same neuron, but overlaps freely with arbors of homologous neurons from adjacent ganglia. Kramer and Kuwada (Kramer and Kuwada 1983) observed that growth cones closely approach isoneuronal branches (often a distance less than the length of a filopodium), but never grow over them. This observation led them to hypothesize that axon branches directly compete for territory. To test this hypothesis, Kramer and Stent (Kramer and Stent 1985) crushed growth cones in developing arbors, thereby delaying or eliminating the development of specific axon subfields (Fig. 1C). This operation resulted in a reduction in the size of the targeted subfield and a compensatory increase in the size of neighboring subfields, supporting the idea that the presence of one arbor inhibits growth of neighboring arbors into the same field.

Self-avoidance is likewise observed among sister dendrites in the insect larval sensory system. In the peripheral nervous system (PNS) sensory neurons with characteristic positions and morphologies innervate the epidermis. The multidendritic-dendritic arborization (da) sensory neurons occupy a two-dimensional space sandwiched between the epidermis and body wall musculature. The da neurons have been segregated based on their morphology, which might also reflect distinct functions (Grueber et al. 2002). The dendrites of all these neurons show isoneuronal self-avoidance (Grueber and Truman 1999; Grueber et al. 2002; Sweeney et al. 2002), but the dendrites of different classes of cells can overlap extensively (Grueber et al. 2001; Grueber et al. 2002). Consistent with the mutual repulsion hypothesis, lesion of single da neuron branches allows the growth of neighboring branches into the vacated area (Sugimura et al. 2003).

Growth cone behaviors consistent with contact-mediated repulsion between isoneuronal branches have been observed by time-lapse imaging in many types of developing arbors, including the dendritic processes of retinal ganglion cells in culture (Montague and Friedlander 1991), cerebellar Purkinje cells in vivo (Sdrulla and Linden 2006), and zebrafish somatosensory neuron axon arbors growing in the skin (Liu and Halloran 2005; Sagasti et al. 2005). Other strategies for minimizing isoneuronal branch overlap are possible, such as competition for a limiting trophic factor or an activity dependent mechanism, but a contactmediated mechanism of self-repulsion is well-supported by both cellular and molecular studies (see below).

\section{MECHANISMS OF SELF-AVOIDANCE}

The findings that isoneuronal branches or subfields actively exclude one another provided an explanation for the exquisite spacing of arbors across their field but raised the question of how growth cones can distinguish "self" (which they avoid), from "other" (which they do not). Self-recognition could be achieved by a molecular code, wherein each neuron expresses distinguishing cell surface molecules, or by another property that would differ between, but not within, cells, such as correlated electrical activity. Wang and Macagno devised a set of branch severing experiments to attempt to distinguish between these two possibilities (Wang and Macagno 1998). They detached a branch of a leech mechanosensory axon with a laser microbeam, which persisted for many hours as a dynamic, and apparently healthy, axon fragment, and observed that neighboring branches grew into the territory occupied by the detached branch. In contrast, self-avoidance seemed to persist among the branches within the detached axon. Thus, in this system cytoplasmic continuity appears to be required for self-recognition. One possibility is that axonal branch correlated activity might be used to discriminate self from nonself in these neurons, although other mechanisms are not excluded (Wang and Macagno 1998).

Molecular studies have focused on cell adhesion molecules as mediators of self versus nonself discrimination. Cellular studies of 
self-avoidance imply that any underlying molecular mechanism must enforce robust and selective recognition only between sister branches, and must link recognition to changes in growth cone behavior (either stopping or repulsion). Studies in Drosophila demonstrate that a highly molecularly diverse cell adhesion molecule can function in self-avoidance. Down syndrome cell adhesion molecule 1 (Dscam1) encodes an immunoglobulin (Ig) superfamily member, which in Drosophila can generate up to 19,008 proteins with distinct ectodomains (Schmucker et al. 2000). In binding assays, Dscams show isoform-specific homophilic interactions, but little interaction occurs between different, yet closely related, isoforms (Wojtowicz et al. 2004; Wojtowicz et al. 2007). Binding specificity arises from an $\mathrm{S}$ shape conformation of the ectodomain that allows each of the variable Ig domains to match with those of an opposing, antiparallel, Dscam1 molecule (Meijers et al. 2007; Sawaya et al. 2008) (Fig. 2).

Several studies have implicated Drosophila Dscam 1 in dendritic and axonal self-avoidance and process spacing in diverse neuronal populations, including mushroom body axons, olfactory projection neuron $(\mathrm{PN})$ dendrites, and da neuron dendrites (Wang et al. 2002; Zhan et al. 2004; Zhu et al. 2006; Hattori et al. 2007; Hughes et al. 2007; Matthews et al. 2007; Schmucker 2007; Soba et al. 2007; Millard and Zipursky 2008) (Fig. 2). Loss of Dscam1 in these neurons leads to a failure in branch separation, which manifests either as fasciculation of processes, collapse of processes into bundles, or process overlap. Single arbitrary isoforms expressed in single neurons can rescue these defects (Zhan et al. 2004; Zhu et al. 2006; Hughes et al. 2007; Matthews et al. 2007; Soba et al. 2007). In contrast, if a single Dscam1 isoform is expressed in populations of neurons whose dendrites normally overlap, their arbors segregate from one another and produce an aberrant tiling-like arrangement (Hughes et al. 2007; Matthews et al. 2007; Soba et al. 2007) (Fig. 2). These data fit with a model in which neurons with overlapping processes express predominantly distinct Dscam1 isoforms, which allow arbors to coexist in space. Manipulation of the Dscam1 locus to restrict the number of potential isoforms to $12,24,576$, and 1,152 , and analysis of deficiency strains with 4,752 potential isoforms, revealed that the size of isoform pool required for robust discrimination between self and nonself was in the thousands for several different populations of neurons tested (Hattori et al. 2009). Given this substantial number of isoforms to choose from, a sufficient number of distinct identities are possible among intermingling neural processes (Neves et al. 2004; Zhan et al. 2004; Zipursky et al. 2006; Hattori et al. 2009).

Vertebrate Dscam, and the closely related Dscam-like1 (DscamL1), are not highly spliced like insect Dscam1. Nevertheless, their function is important for the spacing of cells and neurites in the retina (Fuerst et al. 2008; Fuerst et al. 2009). Dscam mutations lead to aberrant bundling of neurites (both within and between cells) and uneven spacing of cell bodies of bNOS and dopaminergic amacrine cells (Fuerst et al. 2008). Because the processes from neighboring dopaminergic amacrine cells show extensive overlap and make only minor adjustments in field size when present in excess numbers, repulsive signaling is probably not critical for their normal spacing (Keeley and Reese 2009). Dscam may therefore ensure selfavoidance by counterbalancing the action of other adhesion systems that would otherwise lead to the fasciculation of processes (Fuerst et al. 2008; Fuerst and Burgess 2009). This role is supported by studies of retinal ganglion cells (RGCs), most of which express Dscam early during their development and rely on Dscam to prevent cell-type specific fasciculation (Fuerst et al. 2009). It is notable that the function of Dscam in both vertebrates and invertebrates is context and species-dependent, as the molecule has been shown to regulate repulsion, outgrowth, attraction/adhesion, and synapse formation in different systems (Andrews et al. 2008; Fuerst et al. 2008; Ly et al. 2008; Yamagata and Sanes 2008; Li et al. 2009; Liu et al. 2009). In mouse RGCs, however, self-avoidance is a primary defect in Dscam mutant retinas, whereas synaptic specificity appears intact (Fuerst et al. 2009). 


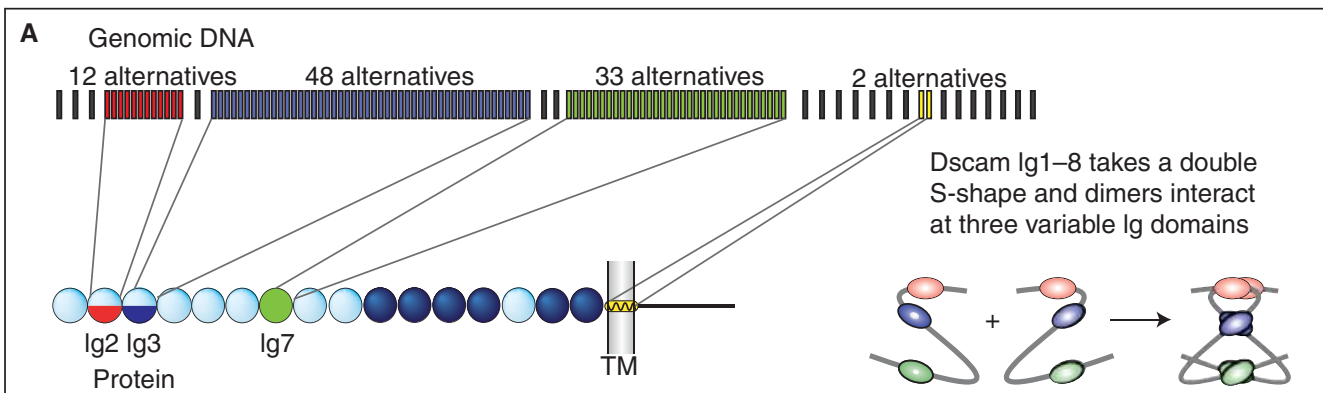

B

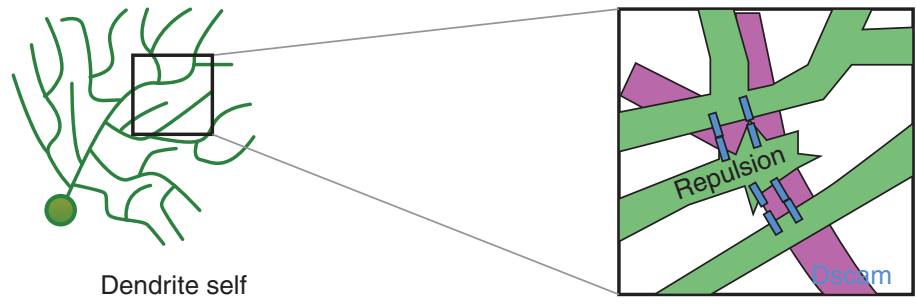

avoidance

C

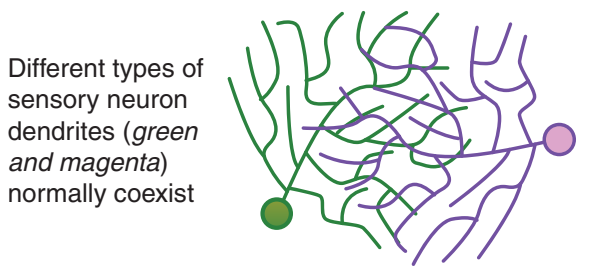

D

Different types of sensory neuron forced to express the same Dscam isoform do not coexist

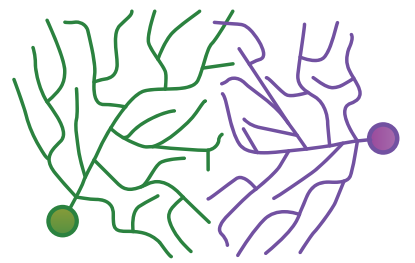

Figure 2. Dscam regulates dendrite self-avoidance. (A) The Dscam locus can generate up to 152,064 distinct protein isoforms by alternative splicing, including 19,008 distinct extracellular domains (Schmucker et al. 2000; Yu et al. 2009). Exons 4 and 6 code for half Ig domains (Ig2 and Ig3 respectively) and exon 9 codes for all of Ig7. Extensive binding assays have shown isoform-specific homophilic binding (Wojtowicz et al. 2004; Wojtowicz et al. 2007). The structure of the homophilic binding region (including Ig1-Ig8) indicates that the protein folds into an $\mathrm{S}$ shaped molecule, and that this folding allows interactions between the three variable Ig domains. Adapted from (Sawaya et al. 2008). (B) Studies in several systems (see text) indicate that Dscam is critical for self-avoidance of dendrites and axons. Studies are consistent with a model in which sister dendrites or axons that encounter one another during development are recognized by virtue of their shared isoform repertoire. This recognition leads to repulsive signaling, the molecular basis of which is not yet understood. $(C)$ Single Dscam isoforms are sufficient for self-recognition and avoidance but Dscam diversity is required between cells so that they can share territories (coexistence). Left: Two different neurons with coexisting arbors. When those cells are forced to express the same arbitrary isoform at high levels their arbors no longer cross each other and are thus unable to coexist (see text for details).

Additional cell surface receptors implicated in self-avoidance include the immunoglobulin superfamily member Turtle, and the Leukocyte Antigen Related (LAR) protein receptor tyrosine phosphatase. Turtle functions in class IV Drosophila da neurons to enforce terminal branch spacing (Long et al. 2009). Studies of the comb cell in leech have identified LAR as a mediator of self-recognition and/or repulsion. Comb cells are transient cells that may serve as a scaffold for muscle cells (Jellies and Kristan 1991). These cells possess numerous parallel extensions with regular spacing between them (Baker and Macagno 2007). A LAR homolog, HmLAR2, is expressed in these cells and knocking down its function with function-blocking antibodies, overexpression of the LAR ectodomain, or RNAi knockdown causes processes to adopt 
irregular trajectories and cross over one another (Baker and Macagno 2000). LAR therefore seems to be a homophilic self-recognition molecule for these cells.

\section{CELLULAR MECHANISMS OF HETERONEURONAL TILING}

Multiple arbors of neurons innervating the same surface are often arranged in a tiled pattern that maximizes coverage of that surface while minimizing overlap between neighboring arbors. Tiling has been studied in several cell types in both vertebrate and invertebrate systems (Fig. 3), revealing common features of the tiling process. For example, contact-mediated mutual repulsion among like neurons is believed to underlie their uniform innervation of a surface by many populations of neurons. Nonetheless, substantial mechanistic diversity is implied by the observation that different cell types innervating a surface tile independently of one another and can vary greatly in the amount of overlap with which they innervate it. Below we survey studies that revealed common and varying features of the tiling process in different model systems.

Neighbor Interactions and Intrinsic Limits Shape Diverse Dendritic Patterns in the Retina

Neurites of each subtype of retinal ganglion cell, horizontal cell, and amacrine cell arborize in one or a few specific laminae of the inner or outer plexiform layer of the retina. The cell bodies of virtually all retinal cell types are arranged as independent, nonrandom mosaics that maximize the distance between neighboring cells (Wassle and Riemann 1978; Rockhill et al. 2000). The arbors of each retinal cell type innervate the retina with widely varying degrees of coverage (Fig. 3A,B). For example, the dendrites of starburst amacrine cells innervate the retina with over 100-fold redundancy (MacNeil and Masland 1998), whereas the dendrites of certain classes of RGCs interlock like jigsaw puzzle pieces, showing almost perfect tiling (Dacey 1993; Amthor and Oyster
1995). Although the regular spacing of cell bodies could help retinal dendritic fields achieve uniform coverage, cell body spacing cannot alone explain the uniformity of dendrite distribution, at least in certain classes of cat retinal ganglion cells (Wassle et al. 1981).

Studies of the mechanisms of retinal tiling suggest that both interactions between neighboring cells and intrinsic determinants help to specify dendritic arbor size and spacing, which in turn impact how evenly arbors tile a surface. Experiments by Perry and Linden (Perry and Linden 1982) provided evidence for interactions among neighboring dendrites (Fig. 3C,D). When they killed RGCs by damaging the optic nerve in rats, effectively denervating a portion of the retina, dendrites of ganglion cells around the denervated region oriented toward it, perhaps indicating attraction toward denervated territory or repulsion by remaining neighboring cells. Hitchcock (Hitchcock 1989) showed a similar phenomenon in goldfish ganglion cells. Although the reorientation of these arbors provided evidence that adjacent arbors influence each other's territories, the dendrites in these experiments never fully re-innervated the denervated territory, perhaps indicating an intrinsic limit in growth potential. Indeed, Montague and Friedlander (Montague and Friedlander 1991) showed that the size and shapes of RGC arbor territories in culture, where they are not directly in contact with neighbors, are similar to those of RGCs in vivo, indicating that arbor size may be intrinsically determined. Lin and Masland (Lin et al. 2004) addressed this issue in vivo by examining two RGC cell types in the retinas of genetic mutants in which fewer RGCs than normal are born or survive. In these heavily depleted retinas, dendritic arbors, which lacked nearby neighbors, were of normal size. Taken together, these studies suggest that these populations of RGCs use a combination of neighbor interactions and intrinsic determinants to specify arbor sizes, probably to different degrees in different neuron classes.

Repulsive interactions may also act transiently to establish territorial domains. During development, horizontal cells extend transient 
A
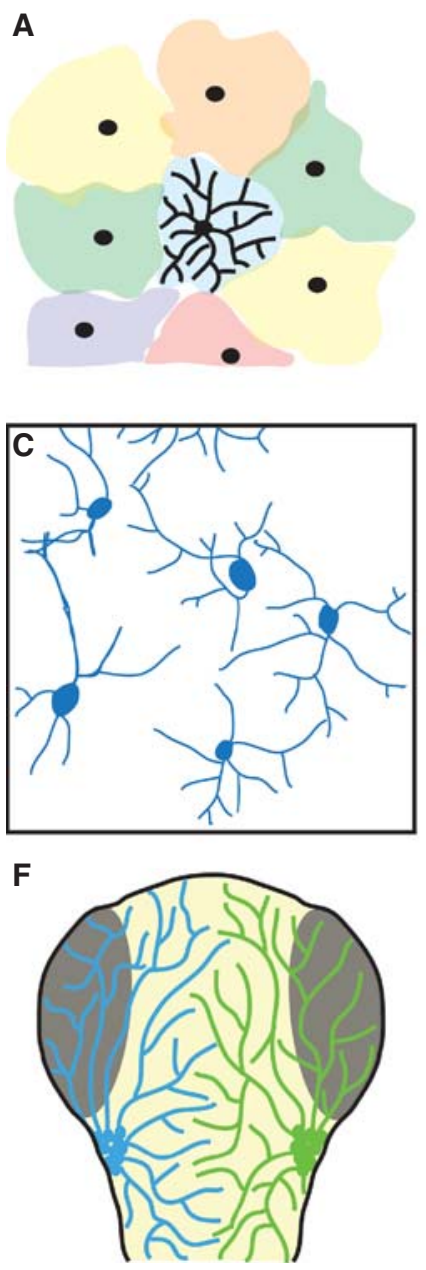

B
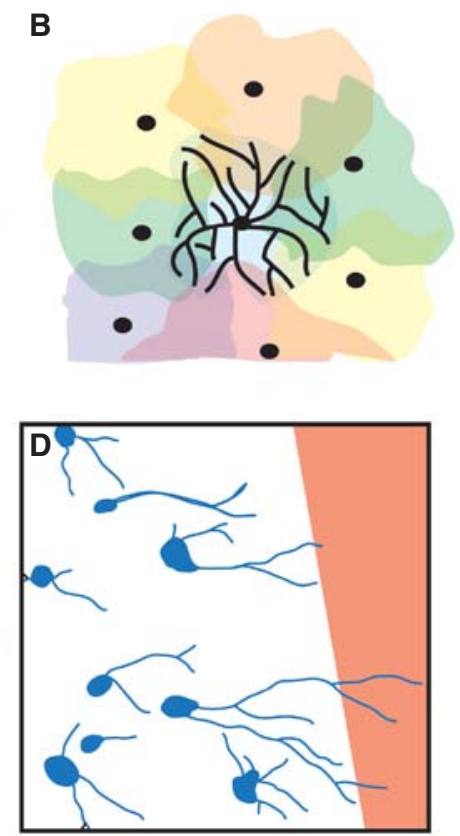

G

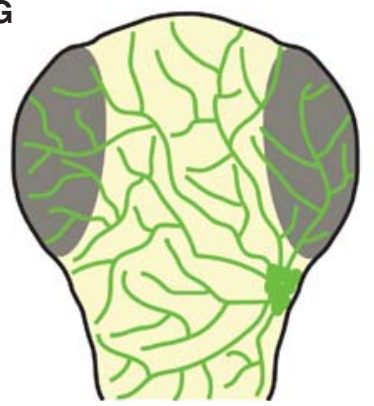

E
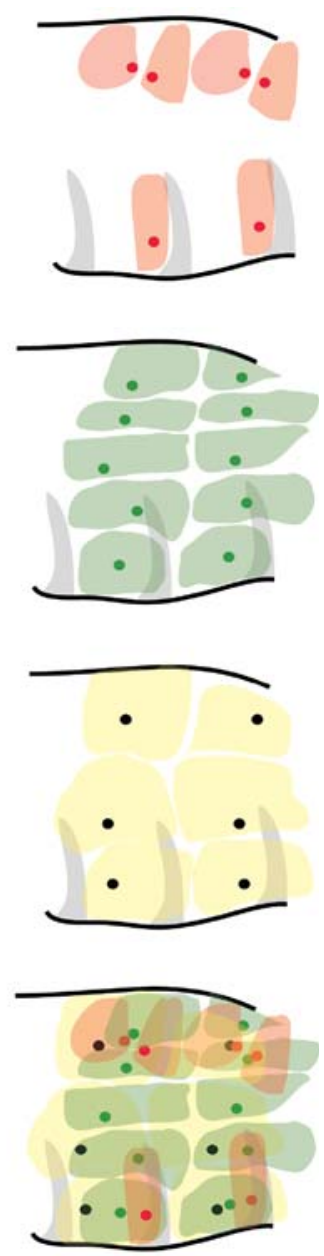

Figure 3. Common features of heteroneuronal tiling. $(A, B)$ Although the cell bodies of all retinal cell subtypes are arranged in a regular mosaic, different subtypes display various degrees of overlap. Colored shapes represent the territories of individual dendrites, black circles represent cell bodies. The dendrite of one cell is diagrammed in each panel. The arbors of some retinal cell types show "perfect" tiling, with closely apposed but nonoverlapping arbors $(A)$, whereas the arbors of other types of neurons extensively overlap $(B)$. Dendritic fields in A are based on images from (Dacey 1993). (C,D) Dendritic arbors of at least some retinal subtypes polarize toward denervated regions. Most ganglion cell dendrites in unperturbed retinas are not polarized in a consistent direction $(C)$, but when a region of the retina is denervated (red area in $D$ ), nearby dendritic arbors polarize toward the empty territory. Images modified from data in (Perry and Linden 1982). (E) Drosophila da neurons exemplify the principle that different neuronal subtypes innervating the same surface are spaced independently of one another. Each diagrams shows two segments of a Drosophila larva, modified from (Grueber et al. 2003). The top panel shows the dendritic fields of one subtype of da neurons that does not uniformly innervate the epidermis. The second and third show the dendritic territories of two different subtypes of da neurons that tile the epidermis almost perfectly. The bottom panel is a superimposition of the territories of the three different neuron subtypes, showing that they each independently innervate the epidermis. $(F, G)$ Somatosensory neurons in embryonic zebrafish show virtually unlimited plasticity. Diagrams represent a dorsal view of the heads of zebrafish larvae. Based on results in (Sagasti et al. 2005). $(F)$. In normal fish, the peripheral axon arbors of the two bilateral trigeminal ganglia are mostly confined to the ipsilateral side of the head. $(G)$ When one trigeminal ganglion is removed early in development, arbors from the remaining ganglion freely cross the midline to innervate the opposite side of the head. 
neurites with nonoverlapping columnar territories (Huckfeldt et al. 2009), even though mature arbors overlap considerably. When cells were killed in developing retinas, neighboring cells rapidly extended these transient neurites to fill the empty space. Thus, transient homotypic repulsion between columnar arbors may play a role in establishing evenly spaced territories by contributing to the creation of retinal mosaics.

Subclasses of Peripheral Sensory Neurons Tile Independently of One Another in Leech and Drosophila

A study of adult leech mechanosensory neurons provided a clear demonstration that functionally distinct neuronal subtypes tile independently at specific boundaries. Three mechanosensory cell types, named $\mathrm{T}$ (touch), $\mathrm{P}$ (pressure), and $\mathrm{N}$ (nociceptive) neurons based on their responses to different intensities of mechanical stimulation, arborize in the skin of adult leech (Nicholls and Baylor 1968). Blackshaw et al. (Blackshaw et al. 1982) killed mechanosensory neurons and electrophysiologically defined the receptive fields of other neurons of both the same and different subtypes. When $\mathrm{N}$ sensory neurons were killed, the receptive fields of remaining $\mathrm{N}$ cells expanded, but not those of T or P cells. Conversely, when $\mathrm{T}$ cells were killed, other $\mathrm{T}$ cells, but not $\mathrm{N}$ or $\mathrm{P}$ cells, expanded their receptive fields. These experiments provided evidence that mechanosensory axons in adult leech retain subtype specific receptive field plasticity.

Different types of neurons innervating the same surface can use distinct strategies for spacing arbors (Fig. 3E). Two classes of da neurons tile the epidermis comprehensively, but independently, of one another, whereas the arbors of two other classes innervate the epidermis sparsely with nonadjacent arbors (Grueber et al. 2002). Killing groups of neurons, as well as single neurons that tile with neighboring neurons, allowed growth of neighboring liketype neurons into the vacated territories (Gao et al. 2000; Grueber et al. 2003; Sugimura et al. 2003). Thus, dendrite-dendrite interactions limit growth and ensure that territories do not overlap in this system. Genetic manipulation of cell identity can produce supernumerary sibling da neurons (Moore et al. 2002). When one class of tiling neuron was overproduced, the extra cells integrated into the tiling pattern (Grueber et al. 2003). In contrast, neurons that show selfavoidance, but do not tile, overlapped almost completely with their duplicated sibling (Grueber et al. 2003). These studies indicate that different classes of neurons possess different capacities for homotypic repulsion.

\section{Neighbor Interactions Limit Vertebrate Somatosensory Axon Territories}

Vertebrate peripheral sensory neurons, including neurons of the trigeminal and dorsal root ganglia (DRG), as well as Rohon-Beard neurons in fish and amphibians, innervate the skin to mediate somatosensation. Each of these neurons extends a peripheral axon that elaborates a terminal arbor in a discrete region of the skin, sometimes called a "dermatome." Experiments in frogs and chicks showed that after surgical removal of an entire DRG normally providing all the sensory innervation to a particular limb, neurons of a neighboring DRG invaded that limb, at least partially restoring sensation (Miner 1956; Frank and Westerfield 1982; Scott 1984). These experiments showed that DRG neurons compete with one another for targets. Because these surgeries were performed before axons reached the skin, competition likely occurs at an early stage of development, perhaps for routes to the periphery.

Competition for territory also occurs among the cutaneous axon terminals of peripheral sensory neurons. The axon arbors of neurons in the two bilateral trigeminal ganglia of larval fish and frogs project two-dimensional arbors that innervate the skin of the head as "free endings." Live time-lapse imaging of growing peripheral axons in zebrafish showed that they repel one another on contact (Liu and Halloran 2005; Sagasti et al. 2005). Normally, the peripheral arbor territories of axons emanating from each trigeminal ganglion are confined to the ipsilateral side of the head, but removing one ganglion allowed axons from the remaining ganglion to project extensively across the midline in 
both fish and frogs (Fig. 3F,G) (Kitson and Roberts 1983; Sagasti et al. 2005). When single neurons were transplanted into neurogenin-1deficient fish, which have no endogenous somatosensory neurons, their peripheral axons expanded to encompass almost the entire head (Sagasti et al. 2005). Unlike retinal dendrites, therefore, axons of zebrafish somatosensory neurons do not seem to possess intrinsic limits to their growth. Moreover, when Rohon-Beard neurons, which normally innervate the trunk and tail, were transplanted into the head they elaborated arbors that innervated the head just like trigeminal neurons. Thus, peripheral sensory neurons are not specified to innervate a particular dermatome in zebrafish larvae; rather, axon arbors simply grow until they encounter a like neighbor. This simple epigenetic strategy allows axons of trigeminal and Rohon-Beard neurons to tile the entire epidermis in an efficient and functionally appropriate manner.

\section{Homotypic Interactions Contribute to Axon} Tiling in the Drosophila CNS

The Drosophila adult eye is a striking example of an ordered arrangement of sensory inputs, with the retina consisting of a crystalline lattice of approximately 700 ommatidial units (Clandinin and Zipursky 2002). Each ommatidium consists of 8 photoreceptor (R) neurons that project axons to second-order processing centers in the lamina (R1-R6) and the deeper medulla layer (R7 and R8) (Fig. 4). In the lamina, R1-R6 axons from a photoreceptor bundle separate from one another to project to an appropriate cartridge. Each cartridge comprises a pool of R cell inputs from several ommatidia that "see" the same point in space. The trajectory taken by axons as they separate from the photoreceptor bundle ultimately affects which postsynaptic lamina neurons each contacts (Chen and Clandinin 2008). R7 and R8 axons bypass this target and restrict their axon terminals to a series of discrete columns within the medulla (Fig. 4). Axon branches do not invade neighboring columns occupied by other R7 or R8 terminals. If extra R7 neurons are generated within a single ommatidium, axons will send collaterals that invade neighboring fields, so long as those fields are empty of other R7s (Ashley and Katz 1994). Thus, for R7 neurons, homotypic interactions are at least one of the cues that shape terminal spacing. A group of neurons that reside in the lamina also project axons that tile within specific medulla layers (Millard et al. 2007), and molecular analyses (see below) likewise implicate homotypic interactions in tiling. Different photoreceptor axons and their targets thus show precise spacing patterns that ensure coherent information transfer in the visual circuit.

\section{MOLECULAR MECHANISMS OF TILING}

Diverse Molecular Mechanisms Regulate Axon Spacing in the Fly Visual System

In the fly visual system, different types of neurons appear to use distinct molecular mechanisms to control spacing with like type cells. Specifying the trajectory of R1-R6 axons is a first step in spacing these axons into distinct cartridges, and is mediated by Flamingo (Fmi)-dependent interactions among axons in the same photoreceptor bundle (Chen and Clandinin 2008) (Fig. 4). Fmi is a seven transmembrane cadherin that can bind homophilically and is expressed throughout R1-R6 neurons (Chae et al. 1999; Usui et al. 1999; Chen and Clandinin 2008). Mosaic knockout analysis indicated that R1-R6 neurons lacking Fmi displayed no obvious defects in axon trajectory or targeting to the appropriate cartridge (Chen and Clandinin 2008). In contrast, neurons adjacent to fmi mutant clones adopted aberrant trajectories, with the severity of the mistargeting decreasing the further the neuron was from the mutant clone (Chen and Clandinin 2008). These results could be explained by a novel balanced opposition model, in which the growth cones of R1-R6 neurons assess the relative level of Fmi signaling coming from either side of the growth cone and pick a trajectory based on balanced pushing or pulling forces generated by Fmi-mediated adhesion (Chen and Clandinin 2008)(Fig. 4). 
W.B. Grueber and A. Sagasti

A

$$
\begin{aligned}
& \text { Motion detection } \\
& \text { photoreceptors (R1-R6) }
\end{aligned}
$$

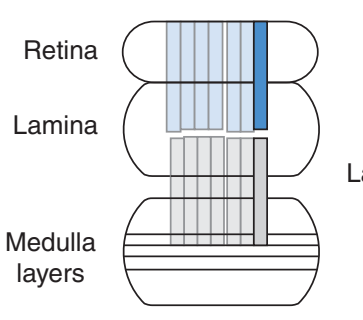

Lamina neurons L1-L5
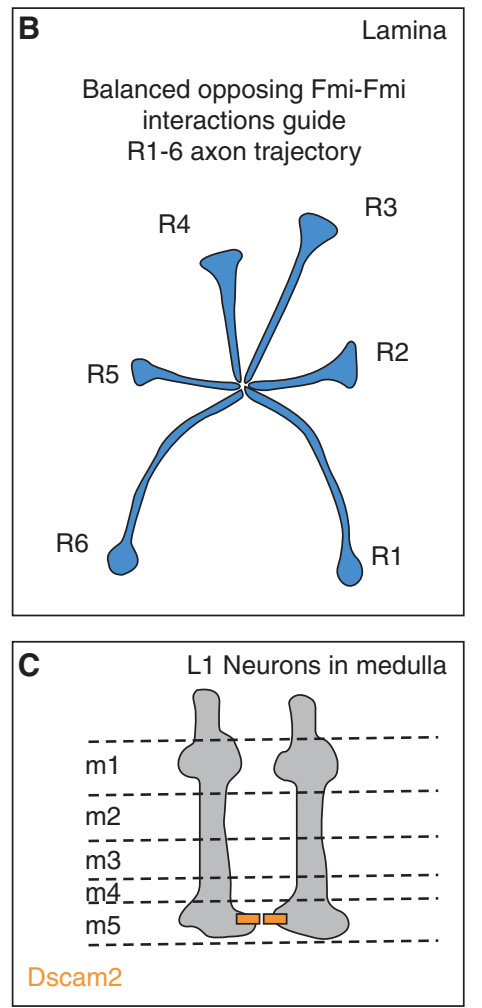

Color vision photoreceptors (R7-R8)

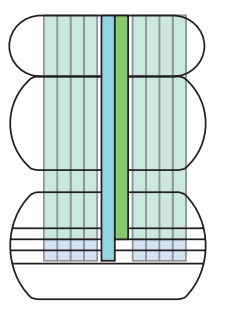

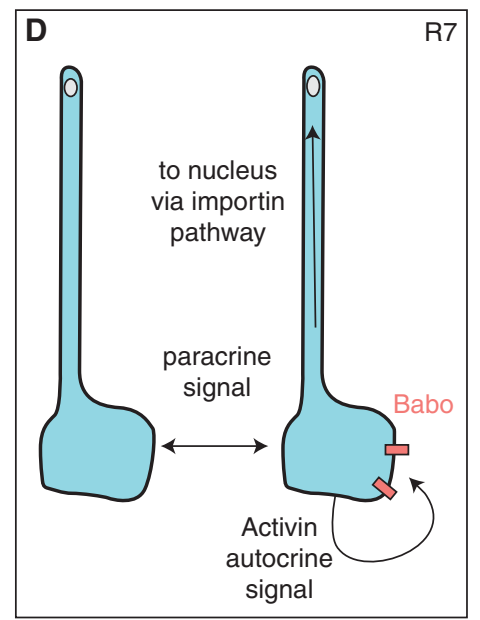

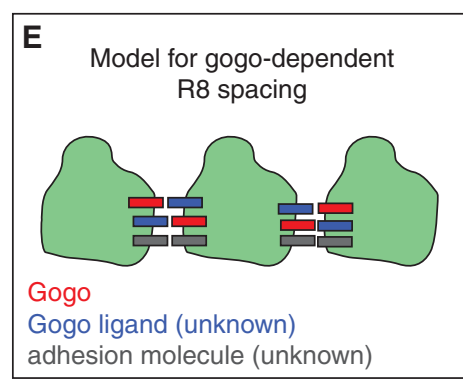

Figure 4. Diverse strategies for process spacing in the fly visual system. (A) Schematic of visual circuitry in Drosophila. (Left) Bundles of R1-R6 photoreceptors extend from the retina to the first order relay in the lamina. Here, the axons split and project in stereotyped directions to lamina cartridges. Each lamina cartridge is comprised of $\mathrm{R}$ neurons that carry visual information from a single point in space. L1-L5 neurons each project to the specific synaptic layers in the medulla neuropil. (Right) Color vision photoreceptors R7 and R8 project from the retina directly to the medulla, bypassing the lamina relay. R7 axons (light blue) project to a deeper medulla layer called $\mathrm{m} 6$, whereas $\mathrm{R} 8 \mathrm{~s}$ (green) project to a more superficial medulla layer (m3). (B) R1-R6 bundles separate to take a specific trajectory to their proper lamina cartridge in a Flamingo-dependent manner (Chen and Clandinin 2008). R1-R6 neurons lacking Fmi show no obvious defects, but neurons adjacent to mutant cells do adopt the wrong trajectory. The growth cones of R1-R6 neurons may assess the relative level of Fmi signaling coming from their neighbors on either side and take a trajectory based on the opposing forces. (C) Dscam2 is essential for tiling of L1 lamina neuron axons in the medulla. L1 axons are normally restricted to a single medulla column by the action of Dscam2. (See facing page for legend.) 
The correct spacing of R7 axons at their target depends both on interactions between different R7 axons and an autocrine signal that limits axon invasiveness into neighboring columns. The autocrine signal is provided by TGF- $\beta$ /Activin signaling via the Activin receptor Baboon, and importin- $\alpha 3$-dependent nuclear accumulation of $\mathrm{dSmad} 2$, together limiting axon arborization (Ting et al. 2007) (Fig. 4). Turtle (Tutl), an IgSF member (see above), is one component of the parallel mutual repulsion pathway (Ferguson et al. 2009). Columnar restriction of R7 axons is disrupted, and imp- $\alpha 3$ tiling phenotypes enhanced, by mutations in tutl (Ferguson et al. 2009). The finding that multiple pathways ensure tiling in this system raises the possibility that in other systems high tiling fidelity may also be an outcome of multiple, partially redundant cues.

R8 axons likewise interact in their target layers of the medulla to ensure even spacing. The genes flamingo ( $f m i)$ and golden goal (gogo) are important for R8-R8 interactions. fmi mutant R8 axons are irregularly spaced, but nevertheless together cover all of their target field (Senti et al. 2003). Thus, fmi is part of the R8 spacing mechanism, but likely acts with other genes such as gogo. Gogo protein contains a Thrombospondin (Tsp1) and a CUB domain (Tomasi et al. 2008) as well as a conserved extracellular "GOGO" domain (consisting of eight conserved cysteine residues and a $\beta$-strand-rich secondary structure) (Tomasi et al. 2008). Whereas single gogo-mutant R8 axons do not display a phenotype, mutant axons that lie adjacent to one another aggregate. It appears that Gogo in $\mathrm{R} 8$ axons acts primarily to mediate repulsive output in response to a ligand also expressed on R8 neurons (Fig. 4). Even in the absence of functional Gogo in single axons, they would still express the ligand and would repel their neighbors. This R8-R8 repulsive signal probably counteracts or silences the activity of a presumed adhesive pathway that also operates between R8 cells (explaining the clumping of gogo-mutant processes) (Fig. 4) (Tomasi et al. 2008). It is notable that both fmi and gogo not only mediate R8-R8 interactions (by possibly intersecting pathways), but are also critical for interactions between R8 axons and their targets.

\section{Dscam2 Mediates Tiling of Drosophila Lamina Neurons}

As described earlier, Dscam1 is an essential regulator of dendrite and axon self-avoidance. The extraordinary degree of alternative splicing of Dscam 1 transcripts makes it unlikely that two different neurons share the same isoforms. The advantages of stochastic alternative splicing for self-avoidance might be a liability for the regulation of tiling between different cells that require a consistent identity, and indeed studies so far have failed to identify a role for fly Dscam 1 in heteroneuronal tiling interactions. Instead, this role resides at least partially with a related gene, Dscam2, which shares domain organization with Dscam1, but is not highly alternatively spliced (Millard et al. 2007). Dscam2 is essential for tiling of L1 lamina neuron axons in the fly visual system (Fig. 4) (Millard et al. 2007). Whereas L1 axons are normally restricted to a single column in the medulla layer, lack of Dscam 2 in individual axons leads to overgrowth and invasion of neighboring

Figure 4. (Continued) Dscam2 molecules engage in homophilic interactions on the growth cones of L1 axons in $\mathrm{m} 1$ and $\mathrm{m} 5$ (Millard et al. 2007). (D) R7 axon tiling in the medulla is regulated by homotypic interactions and a TGF- $\beta$ /Activin autocrine signal limits axon invasiveness for neighboring columns. The self-limiting Activin signal acts through Baboon (babo), a Type I TGF beta receptor. Babo deficient axons can respond to their neighbors, indicating that a different, as yet unknown signal mediates axon-axon interactions (Ting et al. 2007). (E) golden goal regulates R8 axon spacing. Gogo in R8 axons mediates repulsive output, probably in response to an unknown ligand also expressed on R8 neurons. R8-R8 repulsion via Gogo counteracts or silences the activity of an unknown cell adhesion molecule that would otherwise cause R8 axon clumping. Adapted from (Tomasi et al. 2008). 
columns. Like Dscam1, Dscam2 molecules engage in homophilic interactions. Additionally, lack of Dscam2 leads to nonautonomous overgrowth of neighboring axons (Millard et al. 2007). These first insights into tiling mechanisms suggest that cell surface molecules expressed throughout a population of like-type neurons can mediate reciprocal repulsion of processes.

\section{Tiling Mechanisms in Drosophila da Neurons}

Cell surface receptors that mediate tiling of dendritic processes in da neurons are not yet well-understood. A genetic screen identified mutations that cause dendrite overgrowth, one of which was mapped to fmi (Gao et al. 1999; Gao et al. 2000), a gene discussed earlier in the context of photoreceptor axon targeting. Subsequent studies showed that fmi mutant class IV neurons overlap where they meet at the dorsal midline (Kimura et al. 2006). Thus, it appears that repulsive homotypic interactions between like-type neurons are mediated by Fmi. Insight has been gleaned into intracellular signaling pathways involved in tiling of class IV $\mathrm{da}$ neurons. The tricornered (trc) and furry (fry) genes are expressed in all da neurons and act together to ensure repulsion of branches during tiling, and also to suppress dendritic branching (Emoto et al. 2004). Their actions during process repulsion and branching are separable, as branching, but not repulsion, is tied to Rac1 regulation. Trc is a member of the serine-threonine kinase family, thus is likely part of a signaling cascade that allows dendrite repulsion during tiling, following process recognition by surface receptors. Another component of this cascade is the target of rapamycin complex 2 (TORC2) members TOR, Rictor and Sin1 (Koike-Kumagai et al. 2009). Mutations in this complex cause aberrant dendritic overlap of class IV arbors both within and between cells, and TORC2 is essential for Trc kinase activity.

A role for Trc and Fry in process spacing is conserved, as the C. elegans homologs Sax1 and Sax2 are likewise important for the establishment of tiling of sensory dendrites (Gallegos and Bargmann 2004). However, there are some interesting mechanistic differences between fly and worm. Whereas in Drosophila the tiling boundary is formed when dendrites initially meet and Trc/Fry appear to function at this repulsion step, in worms the ALM and PLM mechanosensory processes initially overlap, but then establish their nonoverlapping fields as the animal grows. The late spacing requires a switch from a rapid to a slow growth phase, and this switch is deficient in Sax1 mutants, leading to aberrant overlap of processes (Gallegos and Bargmann 2004).

\section{MAINTENANCE OF TILING}

Tiling is usually accomplished early in development, but this organization can persist into later stages even amidst significant arbor growth and expansion. This observation raises the question of whether the same principles that establish tiling are utilized throughout development to maintain non-overlapping coverage. Neurons differ greatly in the degree to which they maintain their structural plasticity as they mature, and thus their ability to reorganize after injury. Leech mechanosensory neurons, for example, can expand their receptive fields extensively to compensate for a loss of neighbors in adulthood (Blackshaw et al. 1982). Cat retinal ganglion and vertebrate somatosensory neurons cells, on the other hand, can only respond to an absence of neighbors early in development by reorienting their arbors or innervating a limb, respectively (Frank and Westerfield 1982; Eysel et al. 1985). Similarly, the effect of ablation on the fields of Drosophila da neurons is greatest when the manipulation occurs before adjacent fields set up their tiling boundary, and only minor branches invade open territories if ablations are performed after tiling is established (Grueber et al. 2003; Sugimura et al. 2003).

Remarkably, trigeminal cutaneous axons in middle-aged humans have not completely lost their plasticity: After an entire trigeminal ganglion was surgically transected, the receptive fields of contralateral axons expanded slowly across the midline over the course of years 
(Robinson 1983). The observation that human trigeminal neurons maintain a residual, albeit greatly diminished, growth potential suggests that arbor plasticity may serve a purpose throughout the life of an animal. One potential reason for arbors to retain some structural plasticity is that as a neuron ages and grows, dendrite and axon arbors must grow proportionally to maintain their comprehensive innervation of targets, a process that is likely coupled to the expansion of the tissue substrate. The cellular or molecular mechanisms that regulate residual growth potential and coordination with target tissue growth are only beginning to be explored. In Drosophila sensory neurons, such scaling growth of arbors is mediated by a signal between substrate and neuron. In the absence of the bantam microRNA in underlying epithelial cells, dendrites show late-stage overgrowth, suggesting a bantam-mediated signal is required in substrate cells for scaling growth of the overlying dendrite (Parrish et al. 2009). This study was the first to provide molecular insight into the scaling process, so is there is still much to be learned about how neurites maintain their spatial organization as tissues grow.

\section{CONCLUDING REMARKS}

In this review we have attempted to consolidate classical descriptive and experimental studies of process spacing with more recent studies that shed light on the underlying molecular mechanisms of process recognition and repulsion. Self-avoidance and tiling allow neuronal processes (both axons and dendrites) to continually assess the dynamic positions of their neighbors during development, resulting in fine adjustments in growth cone maneuvering to generate uniform territory coverage. Self-avoidance relies on establishment of surface identities that are shared by all sister branches, and distinct from all nonsister branches. Dendritic or axonal tiling mechanisms, in contrast, permit recognition between different cells of the same type. Studies indicate that several distinct cues can underlie axonal tiling in diverse systems, presumably allowing different neuron types to display distinct capacities for repulsion, and to space themselves in a manner fitting with functional requirements. Experimental studies indicate that neurons can compensate for loss of neighboring cells by growing axons or dendrites into vacated regions. In most cases, however, there seem to be clear sensitive periods for invasion, raising important questions about the molecular nature of these sensitive periods. Uncovering the molecular basis of self-avoidance and tiling signals, and the modulation of these signals to specify arbor density and territory size, should provide valuable general insights into neural circuit assembly and plasticity.

\section{ACKNOWLEDGMENTS}

We thank Maria Gallegos and members of the Sagasti and Grueber labs for critical comments on the manuscript, and Anna Dunaevsky for the Purkinje cell image. Supported by NIH grants NS061908 from NINDS (W.B.G.) and DE018496 from NIDCR (A.S.).

\section{REFERENCES}

Amthor FR, Oyster CW. 1995. Spatial organization of retinal information about the direction of image motion. Proc Natl Acad Sci 92: 4002-4005.

Andrews GL, Tanglao S, Farmer WT, Morin S, Brotman S Berberoglu MA, Price H, Fernandez GC, Mastick GS, Charron F, et al. 2008. Dscam guides embryonic axons by Netrin-dependent and -independent functions. Development 135: 3839-3848.

Ashley JA, Katz FN. 1994. Competition and positiondependent targeting in the development of the Drosophila R7 visual projections. Development 120: 1537-1547.

Baker MW, Macagno ER. 2000. The role of a LAR-like receptor tyrosine phosphatase in growth cone collapse and mutual-avoidance by sibling processes. J Neurobiol 44: 194-203.

Baker MW, Macagno ER. 2007. In vivo imaging of growth cone and filopodial dynamics: Evidence for contactmediated retraction of filopodia leading to the tiling of sibling processes. J Comp Neurol 500: 850-862.

Blackshaw SE, Nicholls JG, Parnas I. 1982. Expanded receptive fields of cutaneous mechanoreceptor cells after single neurone deletion in leech central nervous system. J Physiol 326: 261-268.

Bushong EA, Martone ME, Jones YZ, Ellisman MH. 2002. Protoplasmic astrocytes in CA1 stratum radiatum occupy separate anatomical domains. J Neurosci 22: 183-192.

Campbell RE, Gaidamaka G, Han SK, Herbison AE. 2009. Dendro-dendritic bundling and shared synapses between 
W.B. Grueber and A. Sagasti

gonadotropin-releasing hormone neurons. Proc Natl Acad Sci 106: 10835-10840.

Chae J, Kim MJ, Goo JH, Collier S, Gubb D, Charlton J, Adler PN, Park WJ. 1999. The Drosophila tissue polarity gene starry night encodes a member of the protocadherin family. Development 126: 5421-5429.

Chen PL, Clandinin TR. 2008. The cadherin Flamingo mediates level-dependent interactions that guide photoreceptor target choice in Drosophila. Neuron 58: 26-33.

Clandinin TR, Zipursky SL. 2002. Making connections in the fly visual system. Neuron 35: 827-841.

Dacey DM. 1993. The mosaic of midget ganglion cells in the human retina. J Neurosci 13: 5334-5355.

Emoto K, He Y, Ye B, Grueber WB, Adler PN, Jan LY, Jan YN. 2004. Control of dendritic branching and tiling by the Tricornered-kinase/Furry signaling pathway in Drosophila sensory neurons. Cell 119: 245-256.

Eysel UT, Peichl L, Wassle H. 1985. Dendritic plasticity in the early postnatal feline retina: Quantitative characteristics and sensitive period. J Comp Neurol 242: 134-145.

Ferguson K, Long H, Cameron S, Chang WT, Rao Y. 2009. The conserved Ig superfamily member turtle mediates axonal tiling in Drosophila. J Neurosci 29: 14151-14159.

Frank E, Westerfield M. 1982. The formation of appropriate central and peripheral connexions by foreign sensory neurones of the bullfrog. J Physiol 324: 495-505.

Fuerst PG, Burgess RW. 2009. Adhesion molecules in establishing retinal circuitry. Curr Opin Neurobiol 19: 389-394.

Fuerst PG, Bruce F, Tian M, Wei W, Elstrott J, Feller MB, Erskine L, Singer JH, Burgess RW. 2009. DSCAM and DSCAML1 function in self-avoidance in multiple cell types in the developing mouse retina. Neuron 64: 484-497.

Fuerst PG, Koizumi A, Masland RH, Burgess RW. 2008. Neurite arborization and mosaic spacing in the mouse retina require DSCAM. Nature 451: 470-474.

Gallegos ME, Bargmann CI. 2004. Mechanosensory neurite termination and tiling depend on SAX-2 and the SAX-1 kinase. Neuron 44: 239-249.

Gao FB, Brenman JE, Jan LY, Jan YN. 1999. Genes regulating dendritic outgrowth, branching, and routing in Drosophila. Genes Dev 13: 2549-2561.

Gao FB, Kohwi M, Brenman JE, Jan LY, Jan YN. 2000. Control of dendritic field formation in Drosophila: The roles of flamingo and competition between homologous neurons. Neuron 28: 91-101.

Grueber WB, Truman JW. 1999. Development and organization of a nitric-oxide-sensitive peripheral neural plexus in larvae of the moth, Manduca sexta. J Comp Neurol 404: $127-141$.

Grueber WB, Graubard K, Truman JW. 2001. Tiling of the body wall by multidendritic sensory neurons in Manduca sexta. J Comp Neurol 440: 271-283.

Grueber WB, Jan LY, Jan YN. 2002. Tiling of the Drosophila epidermis by multidendritic sensory neurons. Development 129: 2867-2878.

Grueber WB, Ye B, Moore AW, Jan LY, Jan YN. 2003. Dendrites of distinct classes of Drosophila sensory neurons show different capacities for homotypic repulsion. Curr Biol 13: 618-626.
Hattori D, Chen Y, Matthews BJ, Salwinski L, Sabatti C, Grueber WB, Zipursky SL. 2009. Robust discrimination between self and non-self neurites requires thousands of Dscam1 isoforms. Nature 461: 644-648.

Hattori D, Demir E, Kim HW, Viragh E, Zipursky SL, Dickson BJ. 2007. Dscam diversity is essential for neuronal wiring and self-recognition. Nature 449: 223-227.

Hitchcock PF. 1989. Exclusionary dendritic interactions in the retina of the goldfish. Development 106: 589-598.

Huckfeldt RM, Schubert T, Morgan JL, Godinho L, Di Cristo G, Huang ZJ, Wong RO. 2009. Transient neurites of retinal horizontal cells exhibit columnar tiling via homotypic interactions. Nat Neurosci 12: 35-43.

Hughes ME, Bortnick R, Tsubouchi A, Bäumer P, Kondo M, Uemura T, Schmucker D. 2007. Homophilic Dscam interactions control complex dendrite morphogenesis. Neuron 54: 417-427.

Jellies J, Kristan WBJr, 1991. The oblique muscle organizer in Hirudo medicinalis, an identified embryonic cell projecting multiple parallel growth cones in an orderly array. Dev Biol 148: 334-354.

Keeley PW, Reese BE. 2009. Morphology of dopaminergic amacrine cells in the mouse retina: Independence from homotypic interactions. J Comp Neurol 518: 1220-1231.

Kimura H, Usui T, Tsubouchi A, Uemura T. 2006. Potential dual molecular interaction of the Drosophila 7-pass transmembrane cadherin Flamingo in dendritic morphogenesis. J Cell Sci 119: 1118-1129.

Kitson DL, Roberts A. 1983. Competition during innervation of embryonic amphibian head skin. Proc $R$ Soc Lond B Biol Sci 218: 49-59.

Koike-Kumagai M, Yasunaga KI, Morikawa R, Kanamori T, Emoto K. 2009. The target of rapamycin complex 2 controls dendritic tiling of Drosophila sensory neurons through the Tricornered kinase signalling pathway. EMBO J 28: 3879-3892.

Kramer AP, Kuwada JY. 1983. Formation of the receptive fields of leech mechanosensory neurons during embryonic development. J Neurosci 3: 2474-2486.

Kramer AP, Stent GS. 1985. Developmental arborization of sensory neurons in the leech Haementeria ghilianii. II. Experimentally induced variations in the branching pattern. J Neurosci 5: 768-775.

Li HL, Huang BS, Vishwasrao H, Sutedja N, Chen W, Jin I, Hawkins RD, Bailey CH, Kandel ER. 2009. Dscam mediates remodeling of glutamate receptors in Aplysia during de novo and learning-related synapse formation. Neuron 61: 527-540.

Lin B, Wang SW, Masland RH. 2004. Retinal ganglion cell type, size, and spacing can be specified independent of homotypic dendritic contacts. Neuron 43: 475-485.

Liu Y, Halloran MC. 2005. Central and peripheral axon branches from one neuron are guided differentially by Semaphorin3D and transient axonal glycoprotein-1. J Neurosci 25: 10556-10563.

Liu G, Li W, Wang L, Kar A, Guan KL, Rao Y, Wu JY. 2009. DSCAM functions as a netrin receptor in commissural axon pathfinding. Proc Natl Acad Sci 106: 2951-2956.

Livet J, Weissman TA, Kang H, Draft RW, Lu J, Bennis RA, Sanes JR, Lichtman JW. 2007. Transgenic strategies for 
combinatorial expression of fluorescent proteins in the nervous system. Nature 450: 56-62.

Long H, Ou Y, Rao Y, van Meyel DJ. 2009. Dendrite branching and self-avoidance are controlled by Turtle, a conserved IgSF protein in Drosophila. Development 136: 3475-3484.

Ly A, Nikolaev A, Suresh G, Zheng Y, Tessier-Lavigne M, Stein E. 2008. DSCAM is a netrin receptor that collaborates with DCC in mediating turning responses to netrin-1. Cell 133: 1241-1254.

MacNeil MA, Masland RH. 1998. Extreme diversity among amacrine cells: Implications for function. Neuron 20: 971-982.

Matthews BJ, Kim ME, Flanagan JJ, Hattori D, Clemens JC, Zipursky SL, Grueber WB. 2007. Dendrite self-avoidance is controlled by Dscam. Cell 129: 593-604.

Meijers R, Puettmann-Holgado R, Skiniotis G, Liu JH, Walz T, Wang JH, Schmucker D. 2007. Structural basis of Dscam isoform specificity. Nature 449: 487-491.

Millard SS, Zipursky SL. 2008. Dscam-mediated repulsion controls tiling and self-avoidance. Curr Opin Neurobiol 18: $84-89$.

Millard SS, Flanagan JJ, Pappu KS, Wu W, Zipursky SL. 2007. Dscam 2 mediates axonal tiling in the Drosophila visual system. Nature 447: 720-724.

Miner N. 1956. Integumental specification of sensory fibers in the development of cutaneous local sign. J Comp Neurol 105: $161-170$.

Montague PR, Friedlander MJ. 1989. Expression of an intrinsic growth strategy by mammalian retinal neurons. Proc Natl Acad Sci 86: 7223-7227.

Montague PR, Friedlander MJ. 1991. Morphogenesis and territorial coverage by isolated mammalian retinal ganglion cells. J Neurosci 11: 1440-1457.

Moore AW, Jan LY, Jan YN. 2002. hamlet, a binary genetic switch between single- and multiple- dendrite neuron morphology. Science 297: 1355-1358.

Neves G, Zucker J, Daly M, Chess A. 2004. Stochastic yet biased expression of multiple Dscam splice variants by individual cells. Nat Genet 36: 240-246.

Nicholls JG, Baylor DA. 1968. Specific modalities and receptive fields of sensory neurons in CNS of the leech. J Neurophysiol 31: 740-756.

Ogata K, Kosaka T. 2002. Structural and quantitative analysis of astrocytes in the mouse hippocampus. Neuroscience 113: $221-233$.

Parrish JZ, Xu P, Kim CC, Jan LY, Jan YN. 2009. The microRNA bantam functions in epithelial cells to regulate scaling growth of dendrite arbors in drosophila sensory neurons. Neuron 63: 788-802.

Perry VH, Linden R. 1982. Evidence for dendritic competition in the developing retina. Nature 297: 683-685.

Robinson PP. 1983. Recession of sensory loss from the midline following trigeminal sensory root section: Collateral sprouting from the normal side? Brain Res 259: $177-180$.

Rockhill RL, Euler T, Masland RH. 2000. Spatial order within but not between types of retinal neurons. Proc Natl Acad Sci 97: 2303-2307.
Sagasti A, Guido MR, Raible DW, Schier AF. 2005. Repulsive interactions shape the morphologies and functional arrangement of zebrafish peripheral sensory arbors. Curr Biol 15: 804-814.

Sawaya MR, Wojtowicz WM, Andre I, Qian B, Wu W, Baker D, Eisenberg D, Zipursky SL. 2008. A double S shape provides the structural basis for the extraordinary binding specificity of Dscam isoforms. Cell 134: 1007-1018.

Schmucker D. 2007. Molecular diversity of Dscam: Recognition of molecular identity in neuronal wiring. Nat Rev Neurosci 8: 915-920.

Schmucker D, Clemens JC, Shu H, Worby CA, Xiao J, Muda M, Dixon JE, Zipursky SL. 2000. Drosophila Dscam is an axon guidance receptor exhibiting extraordinary molecular diversity. Cell 101: 671-684.

Scott SA. 1984. The effects of neural crest deletions on the development of sensory innervation patterns in embryonic chick hind limb. J Physiol 352: 285-304.

Sdrulla AD, Linden DJ. 2006. Dynamic imaging of cerebellar Purkinje cells reveals a population of filopodia which cross-link dendrites during early postnatal development. Cerebellum 5: 105-115.

Senti KA, Usui T, Boucke K, Greber U, Uemura T, Dickson BJ. 2003. Flamingo regulates R8 axon-axon and axontarget interactions in the Drosophila visual system. Curr Biol 13: 828-832.

Soba P, Zhu S, Emoto K, Younger S, Yang SJ, Yu HH, Lee T, Jan LY, Jan YN. 2007. Drosophila sensory neurons require Dscam for dendritic self-avoidance and proper dendritic field organization. Neuron 54: 403-416.

Sugimura K, Yamamoto M, Niwa R, Satoh D, Goto S, Taniguchi M, Hayashi S, Uemura T. 2003. Distinct developmental modes and lesion-induced reactions of dendrites of two classes of Drosophila sensory neurons. J Neurosci 23: 3752-3760.

Sweeney NT, Li W, Gao FB. 2002. Genetic manipulation of single neurons in vivo reveals specific roles of flamingo in neuronal morphogenesis. Dev Biol 247: 76-88.

Ting CY, Herman T, Yonekura S, Gao S, Wang J, Serpe M, O'Connor MB, Zipursky SL, Lee CH. 2007. Tiling of r7 axons in the Drosophila visual system is mediated both by transduction of an activin signal to the nucleus and by mutual repulsion. Neuron 56: 793-806.

Tomasi T, Hakeda-Suzuki S, Ohler S, Schleiffer A, Suzuki T. 2008. The transmembrane protein Golden goal regulates R8 photoreceptor axon-axon and axon-target interactions. Neuron 57: 691-704.

Usui T, Shima Y, Shimada Y, Hirano S, Burgess RW, Schwarz TL, Takeichi M, Uemura T. 1999. Flamingo, a seven-pass transmembrane cadherin, regulates planar cell polarity under the control of Frizzled. Cell 98: 585-595.

Wang HJ, Macagno ER. 1998. A detached branch stops being recognized as self by other branches of a neuron. Journal of Neurobiology 35: 53-64.

Wang J, Zugates CT, Liang IH, Lee CH, Lee T. 2002. Drosophila Dscam is required for divergent segregation of sister branches and suppresses ectopic bifurcation of axons. Neuron 33: 559-571. 
W.B. Grueber and A. Sagasti

Wassle H, Riemann HJ. 1978. The mosaic of nerve cells in the mammalian retina. Proc $R$ Soc Lond B Biol Sci 200: 441-461.

Wassle H, Peichl L, Boycott BB. 1981. Dendritic territories of cat retinal ganglion cells. Nature 292: 344-345.

Wojtowicz WM, Flanagan JJ, Millard SS, Zipursky SL, Clemens JC. 2004. Alternative splicing of Drosophila Dscam generates axon guidance receptors that exhibit isoform-specific homophilic binding. Cell 118: 619-633.

Wojtowicz WM, Wu W, Andre I, Qian B, Baker D, Zipursky SL. 2007. Avast repertoire of Dscam binding specificities arises from modular interactions of variable Ig domains. Cell 130: 1134-1145.

Yamagata M, Sanes JR. 2008. Dscam and Sidekick proteins direct lamina-specific synaptic connections in vertebrate retina. Nature 451: 465-469.

Yau KW. 1976. Receptive fields, geometry and conduction block of sensory neurones in the central nervous system of the leech. J Physiol 263: 513-538.
Yu HH, Yang JS, Wang J, Huang Y, Lee T. 2009. Endodomain diversity in the Drosophila Dscam and its roles in neuronal morphogenesis. J Neurosci 29: 1904-1914.

Zhan XL, Clemens JC, Neves G, Hattori D, Flanagan JJ, Hummel T, Vasconcelos ML, Chess A, Zipursky SL. 2004. Analysis of Dscam diversity in regulating axon guidance in Drosophila mushroom bodies. Neuron 43: 673-686.

Zhu H, Luo L. 2004. Diverse functions of N-cadherin in dendritic and axonal terminal arborization of olfactory projection neurons. Neuron 42: 63-75.

Zhu H, Hummel T, Clemens JC, Berdnik D, Zipursky SL, Luo L. 2006. Dendritic patterning by Dscam and synaptic partner matching in the Drosophila antennal lobe. Nat Neurosci 9: 349-355.

Zipursky SL, Wojtowicz WM, Hattori D. 2006. Got diversity? Wiring the fly brain with Dscam. Trends Biochem Sci 31: 581-588. 


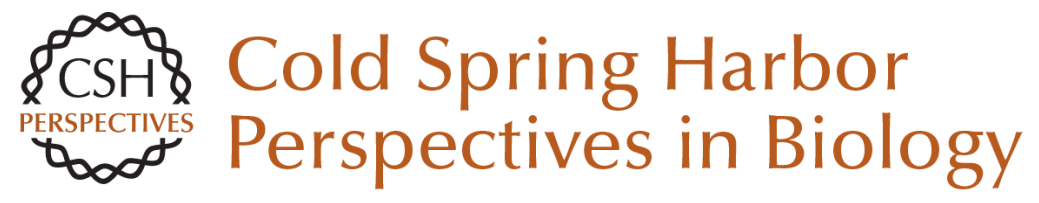

\section{Self-avoidance and Tiling: Mechanisms of Dendrite and Axon Spacing}

Wesley B. Grueber and Alvaro Sagasti

Cold Spring Harb Perspect Biol 2010; doi: 10.1101/cshperspect.a001750 originally published online June 23, 2010

\section{Subject Collection Neuronal Guidance}

Mechanisms and Molecules of Neuronal Wiring: A Primer

Alex L. Kolodkin and Marc Tessier-Lavigne

Guidance Molecules in Axon Pruning and Cell

Death

Pierre Vanderhaeghen and Hwai-Jong Cheng

Initiating and Growing an Axon

F. Polleux and William Snider

Navigating Intermediate Targets: The Nervous System Midline

Barry J. Dickson and Yimin Zou

Cellular Strategies of Axonal Pathfinding

Jonathan Raper and Carol Mason

Guidance Molecules in Axon Regeneration

Roman J. Giger, Edmund R. Hollis II and Mark H. Tuszynski

Signaling from Axon Guidance Receptors Greg J. Bashaw and Rüdiger Klein

Visual Map Development: Bidirectional Signaling, Bifunctional Guidance Molecules, and Competition

David A. Feldheim and Dennis D. M. O'Leary
Wiring the Brain: The Biology of Neuronal

Guidance

Alain Chédotal and Linda J. Richards

Guidance Molecules in Synapse Formation and

Plasticity Kang Shen and Christopher W. Cowan

The Growth Cone Cytoskeleton in Axon

Outgrowth and Guidance

Erik W. Dent, Stephanie L. Gupton and Frank B. Gertler

Topographic Mapping--The Olfactory System Takeshi Imai, Hitoshi Sakano and Leslie B. Vosshall

Self-avoidance and Tiling: Mechanisms of

Dendrite and Axon Spacing Wesley B. Grueber and Alvaro Sagasti

Trafficking Guidance Receptors Bettina Winckler and Ira Mellman

Axon Guidance Molecules in Vascular Patterning Ralf H. Adams and Anne Eichmann

Human Genetic Disorders of Axon Guidance Elizabeth C. Engle

For additional articles in this collection, see http://cshperspectives.cshlp.org/cgi/collection/

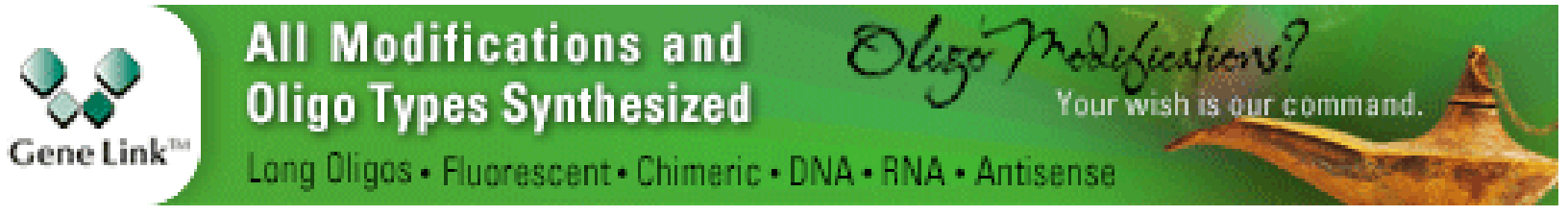

Copyright @ 2010 Cold Spring Harbor Laboratory Press; all rights reserved 